\title{
Infección por SARS-CoV2 en pacientes con trasplante de órgano sólido: Cinco preguntas importantes
}

\author{
SARS-CoV2 infection in patients with solid organ transplant: \\ five important questions
}

Roger Martin', Melibea Sierra-Ruiz²

Cirujano General Universidad Nacional de Colombia. Fellow Cirugía de Trasplante de Órganos Abdominales Universidad ICESI,
Unidad Funcional de Trasplantes Fundación Valle del Lili, Cali, Colombia.
2 Research Fellow, Harvard University, Boston, MA, USA. Centro de Investigaciones clínicas, Fundación Valle del Lili, Cali, Colombia.

\section{Resumen}

Es grande la expectativa que genera en todos los servicios de salud del mundo la rápida expansión del SARSCoV2 (Coronavirus 2 del Síndrome Respiratorio Agudo y Grave), agente etiológico de la Enfermedad Infecciosa por Coronavirus del año 2019, COVID-I9. Por tratarse de una enfermedad emergente es poco lo que se conoce sobre su comportamiento en los humanos, lo que lleva a múltiples interrogantes al momento de tomar decisiones en la práctica clínica. Hasta el momento, las estrategias para enfrentar esta pandemia se basan en la experiencia de los países que han sido epicentro del brote infeccioso y en la evidencia recopilada durante el manejo de otros coronavirus en años anteriores (SARS-CoV en el año 2002 y MERS-CoV en 20I2). La falta de información contundente y unificada ha dado lugar a especulaciones y a suposiciones, especialmente relacionadas con la atención del COVID-I9 en poblaciones consideradas de alto riesgo, como son los pacientes crónicamente inmunosuprimidos postrasplante. A través de esta revisión narrativa de la literatura, más allá de dar la opinión de los autores, se pretende organizar de manera juiciosa los documentos hasta el momento publicados, y responder, basados en datos reales, cinco de las preguntas más importantes que surgen en el día a día durante el manejo de los pacientes trasplantados.

Palabras clave: COVID-I9; virus del SRAS; coronavirus; infecciones por coronavirus; pandemias; cirugía general; trasplante.

Fecha de recibido: 06/04/2020 - Fecha de aceptación: 08/04/2020

Correspondencia: Roger Martin G.

Dirección: Centro de Investigaciones clínicas, Fundación Valle del Lili. Carrera 98 \# 18-49, Cali, Colombia.

Teléfono: 314-4130607. Dirección electrónica: jrmarting@unal.edu.co

Citar como: Martin R, Sierra-Ruiz M. Infección por SARS-CoV2 en pacientes con trasplante de órgano sólido: Cinco preguntas importantes. Rev Colomb Cir. 2020;35:216-26/Especial COVID-19. https://doi.org/10.30944/20117582.623

Este es un artículo de acceso abierto bajo una Licencia Creative Commons - BY-NC-ND https://creativecommons.org/licenses/by-ncnd/4.0/deed.es 


\begin{abstract}
There is a high expectation generated by the rapid expansion of SARS-CoV2 (Severe Acute Respiratory Syndrome related to Coronavirus 2), which is the etiological agent of the Coronavirus Infectious Disease 2019, COVID-I9. As an emerging disease, little is known about its behavior in humans, which generates multiple questions when making decisions in clinical practice. So far, the strategies to face this pandemic are based on the experience of the countries that have been the epicenter of the infectious outbreak and on the evidence collected during the management of other past coronavirus infections such as (SARS-CoV in 2002 and MERS-CoV in 2012). The lack of unified and robust information has given rise to speculation and assumptions primarily related to the care and management of COVID-I9 in populations considered at high risk of infection, such as chronically immunosuppressed patients after transplantation. Beyond giving the opinion from the authors, this narrative review tries to organize the documents published so far and to answer five of the most critical questions that arise every day during the management of transplant patients based on real data.
\end{abstract}

Keywords: COVID-I9; SARS virus; coronavirus; coronavirus infections; pandemics; general surgery; transplantation.

\section{Introducción}

A principios de diciembre de 2019 inició un brote de neumonía en la ciudad de Wuhan, provincia de Hubei en China, y el 3I de diciembre se describió como una infección viral causada por un nuevo tipo de coronavirus nombrado provisionalmente como "novel coronavirus 2019" (2019-nCoV). Estudios taxonómicos posteriores revelaron que se trataba de un beta-coronavirus, filogenéticamente relacionado con el SARS-CoV del año 2002, por lo que fue renombrado como SARS-CoV2 y actualmente se reconoce como el agente etiológico de la Enfermedad Infecciosa por Coronavirus del año 2019 (COVID-I9) '

A pesar de que en las últimas décadas la humanidad se ha enfrentado a varios brotes de neumonías por coronavirus, este nuevo SARS-CoV2, aunque menos mortal que sus antecesores, ha mostrado características epidemiológicas diferentes, siendo un agente mucho más contagioso y de muy rápida expansión. Se ha observado que en un periodo menor a 90 días se ha extendido a más de i8o países y, al momento de escribir este documento (05 de abril de 2020) se han reportado I.273.6I5 casos de contagio con 69.447 muertes confirmadas (tasa de mortalidad de I.6 \%) ${ }^{2,3}$.

Esta rápida expansión y la alta transmisibilidad, motivó a la Organización Mundial de la Salud (OMS) a llamar a un estado de emergencia de salud pública de importancia internacional y a declarar la Pandemia el II de marzo de $2020{ }^{4}$. Colombia es hoy el tercer país más afectado de la región con 1406 casos confirmados y 32 muertes; cifras que han venido creciendo por lo cual se proyecta un aumento de casos en las tres semanas siguientes, alcanzado el pico de contagio hacia la cuarta semana de abril y primera semana de mayo $2020^{5}$.

Uno de los puntos de mayor interés en esta pandemia por SARS-CoV2 ha sido entender el curso natural de la enfermedad; la forma de contagio, el tiempo de incubación, la transmisibilidad, la presentación clínica y, por supuesto, los factores que puedan aumentar el riesgo de contagio y deteriorar el pronóstico de la enfermedad. Con relación a esto último, gracias a los reportes globales de la OMS y a los análisis de datos locales de China, Italia, España y del crucero Diamond Princess ${ }^{6}$, se ha definido que la edad, aunque es el factor de riesgo más importante, no es el único que define la severidad del cuadro clínico sino que existen otros criterios adicionales que en un momento dado definen el curso de la enfermedad, incluso en la población joven. Hoy en día se sabe que antecedentes como la diabetes, la hipertensión arterial, la obesidad, las enfermedades cardiovasculares, las enfermedades pulmonares, el tabaquismo y marcadores clínicos como el puntaje de SOFA elevado y dinero D mayor a Iug/mL, son factores que aumentan el 
riesgo de severidad y mortalidad durante el curso del COVID-19 ${ }^{7}$. Existe una condición adicional que merece especial atención y es el estado de inmunocompetencia al momento de la infección. Aunque los Centros para el Control y Prevención de Enfermedades (CDC) en Atlanta mantienen una alerta sobre las personas con cualquier grado de inmunosupresión que se infecten con SARS$\mathrm{CoV}_{2}$, hasta el momento no es concluyente que este sea un factor de severidad en el COVID-I9.

A continuación, se realizará una revisión de la literatura enfocada específicamente en los pacientes inmunosuprimidos postrasplante de órgano sólido. Colombia ha realizado en las últimas dos décadas aproximadamente $\mathrm{I} 8600$ trasplantes y cuenta en la actualidad con más de I400o pacientes que reciben algún tipo de terapia inmunosupresora postrasplante ${ }^{8}$. La mayoría de ellos, gracias al estricto control médico al que deben someterse mes a mes, se encuentran confinados en las grandes ciudades del país, justo en los sitios en donde se ha presentado el mayor número de contagios y de casos fatales por COVID-I9.

Teniendo en cuenta la obligatoria convivencia entre pacientes trasplantados y casos de SARS$\mathrm{CoV} 2$, existe una alta probabilidad de que en las próximas semanas y durante el pico de contagio se empiecen a presentar casos de COVID-I9 en pacientes con inmunosupresión postrasplante. Por lo anterior se hace indispensable analizar, a la luz de los datos hasta el momento reportados en esta pandemia, cuál es el riesgo real de los pacientes trasplantados y cuál ha sido el comportamiento clínico en los casos infectados en este grupo poblacional. La información se presentará en cinco preguntas importantes, correlacionadas entre ellas, cuyas respuestas pretenden aportar un poco más al conocimiento de esta pandemia.

¿El estado de inmunosupresión postrasplante es un factor de riesgo de contagio del SARS$\mathrm{CoV} 2$ ?

No. El estado de inmunosupresión de un paciente con trasplante de órgano sólido no predispone un riesgo adicional al que tiene la población general para contraer una infección por SARS$\mathrm{CoV} 2$. Mucho se ha escrito sobre la ruta de transmisión y el riesgo de contagio por SARS-CoV2. $\mathrm{El}$ riesgo inicialmente descrito fue el haber estado en contacto con animales silvestres en el "Huanan Seafood Wholesale Market" en Wuhan, Hubei-China. Esta observación se dio a conocer por el hecho de que el $73 \%$ de los pacientes con COVID-I9 infectados en el brote inicial habían visitado dicho mercado ${ }^{9}$.

Hasta ese momento se creía que la transmisibilidad sólo ocurría de animal a humano, siendo el murciélago de cueva del género Miniopterus $s p p$. el hospedero natural de este nuevo betacoronavirus y el Manis pentadactyla (Pangolín Chino) el hospedero intermediario, el cual presenta el virus en cualquiera de sus secreciones. Una vez el virus ha infectado al humano, la transmisión humano-humano ocurre a través de las gotas producidas al toser, estornudar, o incluso al hablar, según informan algunos reportes. En estos escenarios se puede producir un contacto directo con las secreciones o fómites contaminados en los cuales el virus puede durar hasta 9 días, dependiendo del material ${ }^{10}$.

Dada la alta transmisibilidad del SARS-CoV2, investigaciones posteriores han concluido que el contagio se puede dar además por micro-gotas o incluso aerosoles que quedan suspendidos en el aire hasta por tres horas, especialmente en espacios cerrados y cuando se realizan procedimientos invasivos en la vía aérea ${ }^{\mathrm{II}, 12}$. El periodo de incubación varía entre 2-I4 días, con un tiempo promedio de aparición de los síntomas a los 5.I días ${ }^{12,13}$.

Es de especial atención el hecho del contacto inadvertido con una persona infectada que no presente síntomas, ya que hasta el $65 \%$ de los contagios se dan I ó 2 días antes de presentar síntomas. En la casuística China el "Número Básico de Reproducibilidad", llamado " $R-n a u-$ ght" ó "R-zero" (Ro), fue de 2.2 independiente de los factores de riesgo de los contactos, lo que significa que una persona infectada transmitió la infección a 2.2 personas más (sin que existiera prelación por los individuos con estados de 
inmunosupresión) ${ }^{12}$. El Ro más alto reportado hasta el momento ha sido el del crucero Diamond Princess en donde cada persona positiva para COVID-I9 llegó a infectar a 7 personas más. Este es un caso excepcional por tratarse del confinamiento de 37II personas en un mismo barco; a pesar de lo anterior, en dicho informe no se encontró un patrón de riesgo adicional al de la exposición cercana con caso ${ }^{14}$.

En Colombia se ha reportado un Ro de 2.8 y se espera que baje a I.5 con las medidas de aislamiento vital durante la cuarentena (INS). El no usar los elementos de protección personal durante el contacto con una persona infectada por SARS-CoV2 es otro factor de riesgo que amerita especial atención, ya que aumenta significativamente el riesgo de contagio. Entre el personal de salud de Wuhan hubo un $2 \%$ de contagio a pesar de usar todos los elementos de protección personal, a diferencia de España que al momento de este informe presenta un $17 \%$ de contagio entre el personal sanitario por la escases de elementos de protección personal ${ }^{12,15,16}$.

En conclusión, los factores de riesgo para contraer la infección por SARS-CoV2 descritos hasta el momento en la literatura internacional son el contacto cercano con un caso que se encuentre en periodo asintomático o con un caso sospechoso, probable o confirmado, sin los elementos de protección personal; el estado de inmunosupresión no otorga un factor de riesgo adicional.

¿La presentación clínica y la severidad del COVID-I9 son mayores en el paciente inmunosuprimido?

No. El estado de inmunosupresión crónica de un paciente con trasplante de órgano sólido no cambia la presentación clínica del COVID-I9 ni es un factor de riesgo independiente de mal pronóstico. A pesar de que los Centers for Disease Control and Prevention (CDC), Atlanta, aún no ha levantado la alerta ${ }^{17,18}$, no existe literatura que respalde la creencia de que los pacientes con inmunosupresión postrasplante tengan un mayor riesgo de presentar dificultad respiratoria aguda y severa durante el COVID-I9. Por el contrario, cada vez hay más certeza de que un cierto grado de inmunosupresión (especialmente de la inmunidad innata) disminuiría la respuesta inflamatoria exagerada y la fibrosis subyacente que se presenta a nivel del neumocito durante la infección por SARS-CoV2; de ahí que uno de los pilares con mayor éxito durante el tratamiento de los pacientes críticos sea modular la inflamación con esteroides en dosis inmunosupresora, el uso de inmunomoduladores como la Hidroxicloroquina y la disminución de la tormenta de citoquinas con biológicos inmunosupresores como el Tocilizumab (ver más adelante) ${ }^{19,20}$.

A pesar de que existen unas bases teóricas fuertes que hacen pensar que la inmunosupresión es un factor de severidad, la realidad clínica, los datos basados en la evidencia y la experiencia durante brotes de neumonía por otros coronavirus de años anteriores y la información recopilada hasta el momento durante esta pandemia, reportan lo contrario. Por ejemplo, durante la epidemia por SARS-CoV del año 2002-2003, un virus filogenéticamente similar en un $82 \%$ con el SARS-CoV2 pero mucho más letal $(9.5 \%$ tasa de mortalidad), se identificaron con claridad los factores de riesgo que llevaron a aproximadamente 8096 contagios y 774 muertes en 30 países: el ser cuidador de un paciente infectado, la edad mayor de 65 años, ser de género masculino y tener comorbilidades como obesidad, diabetes, enfermedad cardiovascular, enfermedades pulmonares y enfermedad renal crónica estadio 4 ó 5, fueron los factores de riesgo identificados ${ }^{21,22}$. Así mismo, en la epidemia por MERS-CoV del año 2012 (un virus aún más letal; tasa de mortalidad del $35 \%$ ), los factores de riesgo asociados a mal pronóstico fueron nuevamente la edad avanzada (mayor de 65 años), obesidad, tabaquismo, enfermedades pulmonares y enfermedades cardiovasculares con compromiso de la clase funcional ${ }^{23}$. Ni en los informes por SARS-CoV ni en los de MERS-CoV se reportó el estado de inmunosupresión postrasplante como un factor de riesgo independiente asociado a mal pronóstico.

Hablando específicamente de esta pandemia, los informes recopilados de la experiencia de 
China, Italia, España y Estados Unidos hasta el momento tampoco reportan la inmunosupresión crónica postrasplante como factor de severidad. Al primero de abril de 2020 se encuentran en la literatura I2 reportes de caso de pacientes trasplantados que han sido positivos para COVID-I9. Seguramente existen muchos más, pero esta información no ha sido divulgada. La tabla I resume las características principales de los casos hasta el momento publicados. Al observar cada uno de estos reportes es significativo el hecho que la presentación clínica de la infección por SARS$\mathrm{CoV} 2$ en pacientes con inmunosupresión crónica no difiere a la de la población general (fiebre, tos seca, odinofagia y disnea). También coincide el hecho de que la edad y el género masculino son dos factores de severidad no modificables de mal pronóstico.

Sin embargo, hay un dato llamativo y es el hecho de que los síntomas gastrointestinales se presentan con relativa frecuencia entre los pacientes con inmunosupresión postrasplante adicionalmente a los síntomas típicos ya mencionados (4 de I2 pacientes presentaron síntomas gastrointestinales al ingreso) ${ }^{7,17}$. Aparentemente estos síntomas no son tan frecuentes en la población general ya que, por ejemplo, Guan en una serie de 1099 pacientes de 552 hospitales en China encontró que únicamente el $3.8 \%$ de los pacientes presentó síntomas gastrointestinales ${ }^{17}$. Por lo tanto, y hasta no tener más información disponible en pacientes trasplantados, es prudente no desviar la sospecha clínica de COVID-I9 cuando la presentación clínica está acompañada de diarrea, vómito y/o dolor abdominal.

En conclusión, el estado de inmunosupresión postrasplante no es un factor de riesgo de mal pronóstico ni de severidad del COVID-I9; asimismo, la presentación clínica de los pacientes trasplantados que presentan la infección no difiere a la presentación de la población general, con la salvedad de que la aparición de síntomas gastrointestinales como parte del cuadro clínico inicial, puede ser más frecuente en los pacientes inmunosuprimidos.
¿Se podría decir entonces que el estado de inmunosupresión postrasplante es un factor protector que disminuye el riesgo de dificultad respiratoria aguda y severa durante el COVID-I9?

No. Aunque la inmunosupresión crónica postrasplante no es un factor de riesgo de mal pronóstico durante el COVID-I9, hasta el momento no existe información suficiente para concluir que sea un factor protector y que reduzca el riesgo de presentar el síndrome de dificultad respiratoria aguda y severa por coronavirus. Ya que varios autores han reportado el aparente beneficio de la inmunosupresión en el manejo del COVID-I9, vale la pena conocer en qué se basan las observaciones iniciales que han traído esta propuesta y cuáles son las bases teóricas que han llevado a utilizar la inmunosupresión como parte del tratamiento en el paciente críticamente enfermo por SARS-CoV2.

Los beta-coronavirus son virus con una hebra única de RNA trenzada en sentido positivo (+ssRNA); su tamaño es de aproximadamente $30 \mathrm{~Kb}$ y está protegida en el extremo 5 con una CAP y en el extremo 3 ' con una cola de poliA. Poseen además una cápsula en cuya envoltura presentan dos tipos de proteínas Spike I y Spike 2 (SI y S2), las cuales le dan la apariencia de una corona al ser observados en el microscopio electrónico ${ }^{24}$. Por tratarse de un virus RNA, dentro de su mecanismo tiene la plantilla para codificar su propia transcriptasa reversa, siendo esta, junto con las proteínas $\mathrm{N}$ y E, las moléculas que se amplifican a través de RT-PCR durante el diagnóstico de COVID-I9 ${ }^{25}$.

Una vez el virus ha entrado en contacto con la mucosa de la vía aérea superior en el humano, tiene la capacidad de sobrevivir en las secreciones, en donde inicia su periodo de incubación hasta alcanzar el epitelio de revestimiento de las vías aéreas inferiores y los alveolos pulmonares. Aunque el SARS-CoV2 tiene prelación por los neumocitos tipo II, se ha visto que, a diferencia con otros beta-coronavirus, también compromete de forma significativa los neumocitos tipo I. El mecanismo de entrada es a través de la interac- 
Tabla 1: Reporte de caso de pacientes con trasplante de órgano de sólido positivos para COVID-19.

\begin{tabular}{|c|c|c|c|c|c|c|c|}
\hline & $\begin{array}{l}\text { Género y } \\
\text { Edad }\end{array}$ & $\begin{array}{l}\text { Tipo de } \\
\text { Trasplante }\end{array}$ & $\begin{array}{l}\text { Presentación } \\
\text { clínica }\end{array}$ & $\begin{array}{l}\text { Inmunosu- } \\
\text { presión }\end{array}$ & Manejo & Pronóstico & Referencia \\
\hline \multicolumn{8}{|c|}{ Wuhan, China } \\
\hline \multirow[t]{2}{*}{ Caso 1} & \multirow[t]{2}{*}{$\begin{array}{l}\text { Hombre, } 52 \\
\text { años }\end{array}$} & \multirow[t]{2}{*}{$\begin{array}{l}\text { Renal hace } \\
12 \text { años }\end{array}$} & \multirow[t]{2}{*}{$\begin{array}{l}\text { Fiebre, tos, dolor } \\
\text { abdominal }\end{array}$} & \multirow[t]{2}{*}{$\begin{array}{l}\text { Tacro, MMF, } \\
\text { pred }\end{array}$} & \begin{tabular}{|l|} 
suspender inmunosupresión \\
$\begin{array}{l}\text { Metilprednisolona 40mg bolo } \\
\text { diario }\end{array}$ \\
IVIG 10mg día \\
\end{tabular} & \multirow{2}{*}{$\begin{array}{l}\text { Al día } 9 \text { de } \\
\text { hospitalización } \\
\text { se reinicia la } \\
\text { inmunosupresión } \\
\text { y se da egreso al } \\
\text { día } 13 . \\
\end{array}$} & \multirow[t]{2}{*}{12} \\
\hline & & & & & Interferón-y 500000 ui & & \\
\hline \multicolumn{8}{|c|}{ Madrid, España } \\
\hline \multirow{3}{*}{ Caso 2} & \multirow{3}{*}{$\begin{array}{l}\text { Hombre, } 50 \\
\text { años }\end{array}$} & \multirow{3}{*}{$\begin{array}{l}\text { Renal hace } \\
3 \text { años }\end{array}$} & \multirow{3}{*}{$\begin{array}{l}\text { Fiebre, diarrea, } \\
\text { vómito, dolor } \\
\text { abdominal }\end{array}$} & \multirow{3}{*}{$\begin{array}{l}\text { Tacro, Evero, } \\
\text { pred }\end{array}$} & \begin{tabular}{|l|} 
Suspender Tacro y Evero \\
Lopinavir/Ritonavir $400 / 100$ \\
c/12h \\
Ceftarolina / Meropenem \\
\end{tabular} & \multirow{3}{*}{$\begin{array}{l}\text { Fue intubado el } \\
\text { día } 6 \text {, disminución } \\
\text { de parámetros del } \\
\text { ventilador al día } \\
\text { 9. Estable al día } \\
\text { 12. En plan de } \\
\text { extubación }\end{array}$} & \multirow{3}{*}{13} \\
\hline & & & & & $\begin{array}{l}\text { Hidroxicloroquina } 200 \mathrm{mg} \\
\mathrm{c} / 12 \mathrm{~h}\end{array}$ & & \\
\hline & & & & & Interferón-b 250mcg c/48h & & \\
\hline \multicolumn{8}{|c|}{ Bergamo, Italia* } \\
\hline \multirow{2}{*}{ Caso 3} & \multirow{2}{*}{$\begin{array}{l}\text { Hombre, } 50 \\
\text { años }\end{array}$} & \multirow{2}{*}{\begin{tabular}{|l} 
Corazón \\
hace \\
4 meses
\end{tabular}} & \multirow{2}{*}{$\begin{array}{l}\text { Fiebre, tos. } \\
\text { No disnea }\end{array}$} & \multirow{2}{*}{$\begin{array}{l}\text { Ciclosp, MMF, } \\
\text { pred }\end{array}$} & Suspender MMF & \multirow{2}{*}{$\begin{array}{l}\text { Asintomático día } \\
12\end{array}$} & \multirow{2}{*}{ * } \\
\hline & & & & & Manejo expectante & & \\
\hline Caso 4 & $\begin{array}{l}\text { Hombre, } 64 \\
\text { años }\end{array}$ & $\begin{array}{l}\text { Corazón } \\
\text { hace } \\
4 \text { meses }\end{array}$ & $\begin{array}{l}\text { Asintomático. } \\
\text { Prueba tomada } \\
\text { por ser paciente } \\
\text { en diálisis } \\
\end{array}$ & Ciclosp, Evero & Manejo expectante & Asintomático día 8 & * \\
\hline \multirow{2}{*}{ Caso 5} & \multirow{2}{*}{$\begin{array}{l}\text { Hombre, } 66 \\
\text { años }\end{array}$} & \multirow{2}{*}{$\begin{array}{l}\text { Hígado hace } \\
3 \text { meses }\end{array}$} & \multirow{2}{*}{ Fiebre, diarrea } & \multirow{2}{*}{ Ciclosp, Evero } & $\begin{array}{l}\text { Suspender Evero } \\
\text { Lopinavir/Ritonavir 400/100 } \\
\text { c/12h } \\
\end{array}$ & \multirow{2}{*}{$\begin{array}{l}\text { Seguimiento } \\
\text { hospitalizado hasta } \\
\text { el día 12. O2 por } \\
\text { cánula }\end{array}$} & \multirow[t]{2}{*}{ * } \\
\hline & & & & & \begin{tabular}{|l|} 
Hidroxicloroquina $200 \mathrm{mg}$ \\
c/12h
\end{tabular} & & \\
\hline & Mujer, 60 & Renal hace & Fiebre, tos, & & $\begin{array}{l}\text { AzItromicina } \\
\text { Suspender MMF } \\
\text { Lopinavir/Ritonavir 400/100 } \\
\text { c/12h }\end{array}$ & $\begin{array}{l}\text { Seguimiento } \\
\text { hospitalizado hasta }\end{array}$ & 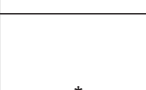 \\
\hline Caso 6 & años & 4 años & disnea & Ciclosp, MMF & \begin{tabular}{|l|} 
Hidroxicloroquina $200 \mathrm{mg}$ \\
$\mathrm{c} / 12 \mathrm{~h}$
\end{tabular} & $\begin{array}{l}\text { el día 4. O2 por } \\
\text { cánula }\end{array}$ & * \\
\hline & & Renal & $\begin{array}{l}\text { Estaba en } \\
\text { manejo de } \\
\text { rechazo humoral }\end{array}$ & & $\begin{array}{l}\text { Suspender inmunosupresión } \\
\text { Lopinavir/Ritonavir 400/100 } \\
\text { c/12h }\end{array}$ & & \\
\hline Caso 7 & $\begin{array}{l}\text { Mujer, } 74 \\
\text { años }\end{array}$ & $\begin{array}{l}\text { hace } \\
29 \text { años }\end{array}$ & $\begin{array}{l}\text { (Rituximab, } \\
\text { plasmaferesis, }\end{array}$ & $\begin{array}{l}\text { Ciclosp, Evero, } \\
\text { MMF }\end{array}$ & $\begin{array}{l}\begin{array}{l}\text { Hidroxicloroquina } 200 \mathrm{mg} \\
\text { c/12h }\end{array} \\
\end{array}$ & Muere al día 5 & * \\
\hline & & & $\begin{array}{l}\text { lgIV) y presenta } \\
\text { fiebre }\end{array}$ & & Azitromicina & & \\
\hline Seattle, $\mathrm{E}$ & EUU** & & & & & & \\
\hline & & Renal & & & Suspender MMF & & \\
\hline Caso 8 & $\begin{array}{l}\text { Hombre, } 54 \\
\text { años }\end{array}$ & $\begin{array}{l}\text { hace } \\
20 \text { años }\end{array}$ & $\begin{array}{l}\text { Fiebre, tos. No } \\
\text { disnea }\end{array}$ & Tacro, MMF & $\begin{array}{l}\text { Hidroxicloroquina } 200 \mathrm{mg} \\
\text { c/12h }\end{array}$ & $\begin{array}{l}\text { Salida a casa } \\
\text { día } 13\end{array}$ & ** \\
\hline Caso 9 & Mujer, 53 & Pulmón hace & Tos disnea & Tacro, AZA, & $\begin{array}{l}\text { Metilprednisolona 50mg día } \\
\text { Inmunosupresión igual }\end{array}$ & Ambulatorio, & $* *$ \\
\hline Caso 9 & años & 20 años & Ios, disnea & pred & Manejo expectante & estable al día 6 & \\
\hline Caso 10 & $\begin{array}{l}\text { Hombre, } 67 \\
\text { años }\end{array}$ & $\begin{array}{l}\text { Hígado hace } \\
19 \text { años }\end{array}$ & $\begin{array}{l}\text { Fiebre, diarrea, } \\
\text { dolor abdominal }\end{array}$ & ciclosp & \begin{tabular}{|l|} 
Inmunosupresión igual \\
Hidroxicloroquina $200 \mathrm{mg}$ \\
c/12h \\
Metilprednisolona $50 \mathrm{mg}$ día \\
\end{tabular} & Salida a casa día 6 & $* *$ \\
\hline & Hombre, 74 & Corazón & Tos, odinofagia, & & Inmunosupresión igual & Ambulatorio, & \\
\hline Caso 11 & años & $\begin{array}{l}\text { hace } \\
23 \text { años }\end{array}$ & fiebre & Tacro & Manejo expectante & estable día 5 & ** \\
\hline Caso 12 & $\begin{array}{l}\text { Mujer, } 55 \\
\text { años }\end{array}$ & $\begin{array}{l}\text { Corazón } \\
\text { hace } \\
13 \text { años }\end{array}$ & $\begin{array}{l}\text { Fiebre, tos, } \\
\text { disnea }\end{array}$ & Tacro & \begin{tabular}{|l|} 
Inmunosupresión igual \\
Hidroxicloroquina $200 \mathrm{mg}$ \\
c/12h \\
Metilprednisolona $50 \mathrm{mg}$ día \\
\end{tabular} & $\begin{array}{l}\text { Observación } \\
\text { hospitalizado } 5 \\
\text { días, salida }\end{array}$ & ** \\
\hline
\end{tabular}

* Datos presentados por el Dr. Attilio Lacovoni, de Heart Transplantation Unit ASST Papa Giovanni XXIII - Bergamo https://www.youtube. com/watch?v=LUM8-vDH-kl\&t=2257s

** Datos presentados por el Dr. Ajit P. Limaye, Transplant Infectious Diseases Program. Seattle, EEUU. https://www.youtube.com/watch?$\mathrm{v}=\mathrm{LUM} 8-\mathrm{vDH}-\mathrm{kl} \& \mathrm{t}=2257 \mathrm{~s}$

Abreviaturas: MMF - micofenolato mofetil, Tacro - tacrolimus, ciclosp - ciclosporina, IVIG - inmunoglobulina intravenosa 
ción de las proteínas Si y S2 con la Enzima Convertidora de Angiotensina tipo 2 (ACE2), que se expresa en su forma no soluble ligada a la membrana del neumocito '. La interacción del virus con el receptor ACE2 es el factor de virulencia más importante ya que desencadena una serie de eventos iniciales que definen el mal pronóstico clínico del paciente. Una vez existe la interacción SI-S2/ACE2, el SARS-CoV2 entra al neumocito, libera su genoma en el ribosoma hospedero y a través del mecanismo de transcripción reversa inicia su replicación formando nuevos viriones maduros y liberándolos, aumentando así la carga viral ${ }^{\text {. }}$.

La carga viral es mayor durante los primeros 7 a Io días, mismo periodo en el cual el individuo es altamente contagioso, aunque se encuentre asintomático. Simultaneo a la replicación viral, la interacción SI-S2/ACE2 disminuye de forma importante la expresión de ACE2 llevando a una acumulación excesiva de angiotensina II y a la activación a nivel pulmonar del eje Renina-Angiotensina-Aldosterona (RAA); lo anterior activa importantes cambios a nivel microvascular como son la vasoconstricción y la remodelación de la membrana alveolo-capilar, disminuyendo la capacidad de intercambio de oxígeno ${ }^{26}$.

Asimismo, la respuesta del sistema inmune innato en contra de la carga viral lleva a un estado de híper inflamación, desatando una tormenta de citoquinas liderada por la exagerada expresión de interleuquina 6 (IL-6) y de todas las proteínas pro-inflamatorias activadas por el factor nuclear $\mathrm{kb}(\mathrm{NFkb})^{27}$. Así las cosas, los cambios vasculares secundarios a la activación del eje RAA y el estado de híper inflamación, no sólo disminuyen el intercambio alveolo-capilar sino que inducen a la remodelación y fibrosis pulmonar ${ }^{26}$. Clínicamente estos cambios moleculares se reflejan en un síndrome de dificultad respiratorio agudo y severo que usualmente se presenta hacia el día 7-8 de la infección, obligando al uso de ventilación mecánica, pronación y todas las demás estrategias de cuidado intensivo encaminadas a recuperar la capacidad de intercambiar oxígeno a nivel alveolo-capilar.
Para evitar llegar a este punto, es importante un estado de inmunosupresión relativa. Teóricamente, al controlar la expresión de proteínas proinflamatorias vía NFkb y bloquear la tormenta de citoquinas liderada por IL-6, será menor la inflamación y la fibrosis alveolar ${ }^{26}$. Estrategias como el uso de Hidroxicloroquina (que disminuye la quimiotaxis de granulocitos y la activación del complemento), de Metilprednisolona (que bloquea NFkb) y de Tocilizumab (inhibidor IL6), podrían tener algún beneficio en el paciente con COVID-I9 y hacen parte hoy de las guías de manejo instauradas en diferentes centros alrededor del mundo ${ }^{19,20}$. Claramente, por ser una enfermedad emergente hace falta recopilar mayor evidencia al respecto, sin embargo, las observaciones clínicas van encaminadas en este sentido.

El beneficio adicional que pueda tener un estado de inmunosupresión crónica con anticalcineurínicos, inhibidores de la m-TOR y/o antimetabolitos todavía es incierto, dado que estos medicamentos no bloquean el sistema inmune innato sino el adaptativo, por tanto, no tendrían ninguna participación directa al momento de bloquear la híper inflamación producida en la etapa más severa del COVID-I9. Pero, al inhibir la activación y proliferación del linfocito "naive" o virgen, bloquean la formación de linfocitos Thi y Th2, lo que finalmente permite tres cosas: I) no existiría daño por citotoxicidad CD8+ en el neumocito, 2) disminuye la disponibilidad de IFN $\gamma$ para la retroalimentación de macrófagos durante la inflamación, y 3) no habría activación del complemento mediada por anticuerpos antígeno-específicos a nivel pulmonar ${ }^{28}$.

En conclusión, la inmunomodulación de la híper inflamación producida a nivel pulmonar durante el COVID-I9 es un pilar fundamental en el tratamiento de la fibrosis y la sepsis por SARS$\mathrm{CoV} 2$. A pesar de que en esta pandemia aún no se ha construido una evidencia clínica significativa, las observaciones iniciales y las robustas bases teóricas respaldan están observación ${ }^{28}$. El aporte que una inmunosupresión crónica postrasplante pueda otorgar a la disminución de la híper inflamación local es incierta, por lo que hasta 
el momento no podemos concluir que el uso de anticalcineurínicos, inhibidores de la m-TOR o antimetabolitos sean un factor protector que disminuya el síndrome de dificultad respiratorio agudo y severo típico del COVID-I9.

¿Se deben quitar los IECAS y los bloqueadores ARA-2 en los pacientes trasplantados que sean positivos para COVID-I9?

No. A pesar de la idea inicial de que los pacientes hipertensos en tratamiento crónico con inhibidores de la ECA (IECAs) y/o bloqueadores del receptor de angiotensina 2 (ARA-2) tenían una súper expresión de ACE2 a nivel pulmonar permitiendo una mayor entrada del SARS-CoV2 al neumocito, hoy existe evidencia suficiente para no recomendar su suspensión ${ }^{26}$.

Como se mencionó en la pregunta anterior, la ACE2 en los humanos se expresa en su conformación no soluble en la membrana de los neumocitos, así como en el riñón y el corazón ${ }^{27}$. Diferentes estudios en humanos han confirmado que a pesar de la similitud molecular entre la ACE y la ACE2, el uso de IECAS no aumenta la expresión de ACE2. De igual manera, en un estudio de cohorte longitudinal realizado en Japón midiendo el impacto de los ARA-2 en la expresión de ACE2 se encontró que únicamente los pacientes que toman Olmesartán de forma crónica presentan elevación de niveles de ACE2 en orina ${ }^{29}$.

Teniendo en cuenta lo anterior, no existe razón para quitar de forma súbita los IECAS o ARA-2 en los pacientes con trasplante de órgano sólido que reciban estos medicamentos. Por el contrario, existe una alerta de diferentes sociedades de cardiología del mundo, en la cual reportan las implicaciones a nivel cardiovascular que puede tener el hecho de suspender los IECAS y/o los ARA-2 durante la infección por COVID-I9; esto podría llevar a inestabilidad del miocardio deteriorando al paciente durante el proceso infeccioso.

Por otra parte, y dejando de lado la idea de que usar los IECA y los ARA-2 son un factor de riesgo de severidad durante el COVID-I9, vale la pena nombrar que, contrario a lo anterior, existen estudios clínicos en curso que pretenden demostrar el efecto benéfico que puede tener el uso de novo de Losartán en los pacientes con COVID-I9. Esto basado en observaciones experimentales de que los ARA-2 cuando no han sido usados antes, podrían ayudar a estabilizar el eje RAA en el pulmón durante la infección por SARS-CoV2 (NCTo4312009).

En conclusión, no es recomendable suspender los IECAS y/o los ARA-2 durante la infección por COVID-I9 ya que estos medicamentos no aumentan la expresión de ACE2 y no tienen ninguna participación en la severidad de la enfermedad. Por el contrario, suspenderlos aumenta el riesgo de eventos coronarios agudos y de falla cardiaca durante la infección. Se debe prestar especial atención a los pacientes que toman Olmesartán, más no a los que toman cualquier otro ARA-2.

¿Teniendo en cuenta la interacción medicamentosa, cual sería el tratamiento recomendado en un paciente con trasplante de órgano sólido positivo para COVID-I9?

Teniendo en cuenta que no existe un tratamiento universalmente aprobado para el COVID-I9 y que la evidencia hasta el momento recopilada es muy débil para recomendar un esquema farmacológico efectivo, a continuación se resumirán los medicamentos que de forma experimental se han utilizado en el tratamiento de los pacientes infectados por SARS-CoV2 y que hoy hacen parte de los protocolos de manejo propuestos en diferentes centros alrededor del mundo:

I. Atazanavir, un antiretroviral utilizado en el tratamiento del VIH/SIDA, está siendo utilizado de forma experimental para el tratamiento del COVID-I9. Por tratarse de un inhibidor de la proteasa del virus, se espera que tenga algún beneficio sobre la replicación del SARS-CoV2. Es metabolizado en un $93 \%$ por la $\mathrm{CYP}_{3} \mathrm{~A} 4$, por lo que presenta interacción medicamentosa aumentando la biodisponibilidad de la Ciclosporina y 
el Tacrolimus, entonces se recomienda una monitorización cercana de sus niveles. Definitivamente no se debe usar en combinación con inhibidores de la m-TOR ${ }^{30}$.

2. Lopinavir, también es un antirretroviral inhibidor de la proteasa utilizado en el tratamiento y la prevención del VIH/SIDA, que siempre se administra de forma combinada Lopinavir / Ritonavir para aumentar su biodisponibilidad. Es metabolizado en un 97\% por el $\mathrm{CYP}_{3} \mathrm{~A}_{4}$ presentando las mismas interacciones medicamentosas que el Atazanavir. Además, existe una alerta sobre la interacción del Lopinavir con el Micofenolato Mofetil, ya que al parecer aumenta su biodisponibilidad promoviendo la mielotoxicidad ${ }^{30,31}$.

3. Remdesivir, un antiretroviral que inhibe la RNA polimerasa viral, empleado en el manejo de la infección por Ébola, también está siendo utilizado en algunos protocolos experimentales en el manejo del COVID-I9. No presenta interacción con ningún medicamento inmunosupresor utilizado en el manejo de los pacientes con trasplante de órgano sólido ${ }^{30}$.

4. Cloroquina e Hidroxicloroquina (ambos comparten el mismo mecanismo de acción y metabolismo), es un medicamento que en Colombia hace parte de los protocolos de tratamiento para COVID-I9 propuestos por el Ministerio de Salud y de la Protección Social. Incluso, hace parte de varios estudios experimentales que pretenden demostrar su beneficio como profilaxis de la infección por SARS-CoV2. La Hidroxicloroquina es en principio un antimalárico, que dadas sus propiedades inmunomoduladoras también ha sido utilizado en el tratamiento de un amplio espectro de enfermedades autoinmunes. Puede ser utilizado en los pacientes con trasplante de órgano sólido que sean positivos para COVID-I9, pero se debe prestar especial atención a los niveles de Tacrolimus, Ciclosporina y los inhibidores de m-TOR, ya que aumenta su biodisponibilidad ${ }^{30,32}$.
5. Tocilizumab, un anticuerpo monoclonal anti-IL6 humana aprobado para el tratamiento de la artritis reumatoidea y más recientemente utilizado en el manejo del rechazo mediado por anticuerpos en el trasplante renal, ha sido propuesto ahora como el pilar fundamental en el control de la tormenta de citoquinas en los pacientes críticamente enfermos con SARS-CoV2 ${ }^{20}$. Se debe tener en cuenta que disminuye la biodisponibilidad de Tacrolimus y de Ciclosporina, siendo necesario un ajuste de la dosis según los niveles. Adicionalmente se debe prestar atención al uso concomitante con Hidroxicloroquina y Micofenolato Mofetil ya que puede aumentar el riesgo de mielotoxicidad.

En conclusión, inicialmente no se recomienda suspender la inmunosupresión en un paciente trasplantado que sea infectado por SARS-CoV2. El tratamiento se debe ajustar a los protocolos institucionales teniendo en cuenta las etapas del espectro de la enfermedad y especial atención se debe prestar a las interacciones medicamentosas descritas anteriormente.

\section{Cumplimiento de normas éticas}

Consentimiento informado: Esta publicación es una revisión de la literatura, y como tal no hay necesidad de un consentimiento informado ni de aprobación del Comité de Ética Institucional.

Declaración de conflicto de intereses: Los autores no declaran ningún conflicto de interés.

Fuentes de financiación: Recursos propios de los autores.

\section{Referencias}

I. Chan JF-W, Kok K-H, Zhu Z, Chu H, To KK-W, Yuan S, et al. Genomic characterization of the 2019 novel human-pathogenic coronavirus isolated from a patient with atypical pneumonia after visiting Wuhan. Emerg Microbes Infect. 2020;9:22I-36.

2. Johns Hopkins coronavirus resource center. COVID-I9 map [cited 2020 Apr 6]. Disponible en: https://coronavirus.jhu.edu/map.html

3. TrackCorona. [cited 2020 Apr 6]. Disponible en: https:// www.trackcorona.live/

4. World Health Organization. WHO Director-General's opening remarks at the media briefing on COVID-I9. 
[cited 2020 Apr 6]. Disponible en: https://www.who. int/dg/speeches/detail/who-director-general-s-opening-remarks-at-the-media-briefing-on-covid-I9---IImarch-2020

5. Ministerio de Salud Colombia. CORONAVIRUS (COVID-I9) [cited 2020 Apr 6]. Disponible en: https://www. minsalud.gov.co/salud/publica/PET/Paginas/Covid-I9_ copia.aspx

6. Russell TW, Hellewell J, Jarvis CI, van-Zandvoort K, Abbott S, Ratnayake R, et al. Estimating the infection and case fatality ratio for COVID-I9 using age-adjusted data from the outbreak on the Diamond Princess cruise ship. medRxiv. 2020 Mar 9;2020.03.05.2003I773. doi: https://doi.org/IO.IIOI/2020.03.05.20031773

7. Zhou F, Yu T, Du R, Fan G, Liu Y, Liu Z, et al. Clinical course and risk factors for mortality of adult inpatients with COVID-I9 in Wuhan, China: a retrospective cohort study. Lancet Lond Engl. 2020 28;395:IO54-62.

8. Instituto Nacional de Salud. Red nacional de donación y trasplante de órganos y tejidos marco legal, documentos técnicos y estadísticas. [cited $2020 \mathrm{Apr}$ 6]. Disponible en: https://www.ins.gov.co/Direcciones/ RedesSaludPublica/DonacionOrganosYTejidos/Paginas/Marco-Legal,-Documentos-Tecnicos-y-Estadisticas.aspx

9. World Health Organization. Novel coronavirus situation report 2 [cited 2020 Apr 6]. Disponible en: https:// www.who.int/docs/default-source/coronaviruse/ situation-reports/20200I22-sitrep-2-2019-ncov.pdf?sfvrsn $=4 \mathrm{~d} 5$ bcbca_2

Io. van Doremalen N, Bushmaker T, Morris DH, Holbrook MG, Gamble A, Williamson BN, et al. Aerosol and surface stability of SARS-CoV-2 as compared with SARS-CoV-I. N Engl J Med. 2020 Mar I7;O(o):null. DOI: IO.IO56/NEJMc2004973

II. World Health Organization. Modes of transmission of virus causing COVID-I9: implications for IPC precaution recommendations [Internet]. [cited $2020 \mathrm{Apr}$ 6]. Disponible en: https://www.who.int/news-room/ commentaries/detail/modes-of-transmission-of-virus-causing-covid-I9-implications-for-ipc-precaution-recommendations

I2. Li Q, Guan X, Wu P, Wang X, Zhou L, Tong Y, et al. Early transmission dynamics in Wuhan, China, of novel coronavirus-infected pneumonia. N Engl J Med. 2020 Mar 26;382:1199-207.

I3. Chan JF-W, Yuan S, Kok K-H, To KK-W, Chu H, Yang J, et al. A familial cluster of pneumonia associated with the 2019 novel coronavirus indicating person-to-person transmission: a study of a family cluster. The Lancet. 2020 Feb I5;395:5I4-23.

I4. Kakimoto K. Initial investigation of transmission of COVID-I9 among crew members during quarantine of a cruise ship - Yokohama, Japan, February 2020. MMWR Morb Mortal Wkly Rep [cited 2020 Apr
6];69. Disponible en: https://www.cdc.gov/mmwr/volumes/69/wr/mm69IIe2.htm

I5. Liu M, He P, Liu HG, Wang XJ, Li FJ, Chen S, et al. Clinical characteristics of 30 medical workers infected with new coronavirus pneumonia. Zhonghua Jie He $\mathrm{He} \mathrm{Hu}$ Xi Za Zhi Zhonghua Jiehe He Huxi Zazhi Chin J Tuberc Respir Dis. 2020 Feb 17;43(0):Eor6.

I6. Clinical characteristics of 138 hospitalized patients with 2019 novel coronavirus-infected pneumonia in Wuhan, China. Critical Care Medicine. JAMA Network. [cited 2020 Apr 6]. Disponible en: https://jamanetwork.com/ journals/jama/fullarticle/276ro44

17. Clinical characteristics of coronavirus disease 2019 in China. NEJM. [cited 2020 Apr 6]. Disponible en: https:// www.nejm.org/doi/full/Io.IO56/NEJMoa2002032

18. Centers for Disease Control and Prevention. Coronavirus disease 2019 (COVID-I9). [cited 2020 Apr 6]. Disponible en: https://www.cdc.gov/coronavirus/2019-ncov/ need -extra-precautions/people-at-higher-risk.html

19. Gauret P, Lagier JC, Parola P, Meddeb L, Mailhe M, Doudier B, et al. Clinical and microbiological effect of a combination of hydroxychloroquine and azithromycin in 80 COVID-I9 patients with at least a sex day follow up: an observational study. [cited 2020 Apr 6]. Int J Antimicrob Agents. 2020 Mar 20:I05949. doi: I0.IOI6/j. ijantimicag.2020.I05949

20. XIaoling Xu, Mingfeng $\mathrm{H}$, Tiantian L, Wei S, Dongsheng W, Binqing F, et al. Effective Treatment of severe COVID-I9 patients with Tocilizumab. [cited 2020 Apr 6]. Disponible en: http://www.chinaxiv.org/ abs/202003.00026vi

2I. Stockman LJ, Massoudi MS, Helfand R, Erdman D, Siwek AM, Anderson LJ, et al. Severe acute respiratory syndrome in children. Pediatr Infect Dis J. 2007 Jan;26:68-74.

22. World Health Organization. Consensus document on the epidemiology of severe acute respiratory syndrome (SARS). [cited 2020 Apr 6]. Disponible en: https://apps. who.int/iris/bitstream/handle/I0665/70863/WHO_ CDS_CSR_GAR_2003.II_eng.pdf

23. Middle East respiratory syndrome coronavirus: risk factors and determinants of primary, household, and nosocomial transmission - The Lancet Infectious Diseases. [cited 2020 Apr 6]. Disponible en: https:// www.thelancet.com/journals/laninf/article/PIISI4733099(I8)30I27-o/fulltext

24. Song W, Gui M, Wang X, Xiang Y. Cryo-EM structure of the SARS coronavirus spike glycoprotein in complex with its host cell receptor ACE2. PLoS Pathog. 20I8;I4(8):eIoo7236.

25. Jin X, Lian J-S, Hu J-H, Gao J, Zheng L, Zhang Y-M, et al. Epidemiological, clinical and virological characteristics of 74 cases of coronavirus-infected disease 2019 
(COVID-I9) with gastrointestinal symptoms. Gut. 2020 Mar 24. http://dx.doi.org/IO.II36/gutjnl-2020-320926

26. Muthiah Vaduganathan, Orly Vardeny, Pharm D, Thomas Michel, John J.V. McMurray, Marc A. Pfeffer, Scott D. Solomon. Renin-Angiotensin-Aldosterone System Inhibitors in Patients with Covid-I9. NEJM. 30 mar 2020. [cited 2020 Apr 6]. https://doi.org/IO.IO56/ NEJMsr200576o

27. Hamming I, Timens W, Bulthuis MLC, Lely AT, Navis GJ, van Goor H. Tissue distribution of ACE2 protein, the functional receptor for SARS coronavirus. A first step in understanding SARS pathogenesis. J Pathol. 2004;203:63I-7.

28. Shi Y, Wang Y, Shao C, Huang J, Gan J, Huang X, et al. COVID-I9 infection: the perspectives on immune responses. Cell Death Differ. 2020 Mar 23;I-4.

29. Campbell DJ, Zeitz CJ, Esler MD, Horowitz JD. Evidence against a major role for angiotensin converting enzyme-related carboxypeptidase (ACE2) in angiotensin peptide metabolism in the human coronary circulation. J Hypertens. 2004;22:197I-6.

30. University Liverpool UK. Liverpool Drug Interactions Group. Interactions with experiemental COVID-I9 therapies. Liverpool, UK. University Liverpool; 2020. Disponible en: https://www.covidı9-druginteractions.org

3I. Cao B, Wang Y, Wen D, Liu W, Wang J, Fan G, et al. A Trial of Lopinavir-Ritonavir in Adults Hospitalized with Severe Covid-I9. N Engl J Med. 2020 Mar I8;o(o):null. DOI: IO.IO56/NEJMoa200I282

32. Gautret P, Lagier J-C, Parola P, Hoang VT, Meddeb L, Mailhe $\mathrm{M}$, et al. Hydroxychloroquine and azithromycin as a treatment of COVID-I9: results of an open-label non-randomized clinical trial. Int J Antimicrob Agents. 2020 Mar 20;105949. 